\title{
IN VITRO STUDY OF LIGHT RADIATION PENETRATION THROUGH DENTIN, ACCORDING TO THE WAVELENGTH
}

\author{
V. Dogandzhiyska ${ }^{1}$, I. Angelov ${ }^{2}$, Sl. Dimitrov $^{3}$ and Ts. Uzunov ${ }^{1}$ \\ ${ }^{1}$ Department of Conservative Dentistry, FDM, MU - Sofia, Bulgaria \\ ${ }^{2}$ Institute of Organic Chemistry, Bulgarian Academy of Sciences, Sofia, Bulgaria \\ ${ }^{3}$ Department of Conservative Dentistry, FDM, MU - Varna, Bulgaria
}

Summary. Based on their biological, analgesic and antibacterial effect, lasers are becoming more widely used in clinical practice in the recent years. In order to obtain a therapeutic effect and to prevent dental pulp alteration in the treatment of hard tooth tissue, the parameters of laser radiation should be precisely controlled. Upon irradiation of dental pulp, these effects are dependent on the permeability of light through the enamel and dentin. The purpose of our study was to examine the penetration of light through dentin, according to the wavelength.The absorption of light was also studied. For the aim of our investigation 30 dentin samples with thickness $1 \mathrm{~mm}$ of extracted human teeth (premolars and molars) were prepared. The absorption and transmission of light in the range $350-1000 \mathrm{~nm}$ in its passage through the samples were studied using a spectrophotometer Ocean Optics QE65000. The obtained results showed that the light absorption of dentin in spectral range 350-600 $\mathrm{nm}$ depends on the wavelength. When light sources, emitting in the wavelength range above $600 \mathrm{~nm}$ were used, the dependence was low. These features should be taken into account in the choice of irradiation wavelength and a light source.

Key words: laser, light penetration, dentin

\section{INTRODUCTION}

$\mathrm{n}$ the dental clinical practice equipments and devices emitting light in a wide spectral range increasingly were used. They were applied in treatment of dental hard tissues, for surgery, physiotherapy, disinfection of infected root canals, etc. [1]. Different effects can be achieved by irradiation of an object treated with light, and responding to different spectral, energy and temporal characteristics 
[2]. In treatment of dental hard tissues lasers were used in the wavelength range 2-3 $\mu \mathrm{m}$ and power in range 8-10 W. For surgery and root canal disinfection (apicoectomy, decontamination) lasers were applied in the visible and infrared spectral region. The most commonly used lasers for surgical procedures in clinical practice are in the wavelength range $800-1100 \mathrm{~nm}$ and a power up to $10 \mathrm{~W}$. In this spectral region the light penetration in biological tissues is high and reaches to $10 \mathrm{~mm}$. In physiotherapy light sources are commonly used in range $630-750 \mathrm{~nm}$, wherein light penetration and influence on the tissue may reach 6-8 $\mathrm{mm}$. By laser-assisted endodontic treatment the light penetrates up to $1 \mathrm{~mm}$ in the dentinal tubules and kills microorganisms, while by conventional treatment with liquid disinfectants the antibacterial effect is quite superficial $[4,5,6]$. When density of light emission increases, the possibility of undesired effects on tissues also increases. These facts require future study of light penetration in the wavelength range from 350 to $1000 \mathrm{~nm}$ through the dentin.

\section{AIM/OBJECTIVE OF THE STUDY}

The aim of our study was to determine the degree of penetration of irradiation of ultraviolet, visible and near infrared spectral regions through dentin of extracted human teeth.

\section{MATERIAL AND METHODS}

For the purpose of our investigation 30 dentine samples with $1 \mathrm{~mm}$ thickness were prepared. All samples were obtained by a transverse section in the area of the pulp chamber roof of extracted human premolars and molars. For cutting diamond burs (Komet, 6859.314.018) and high-speed handpieces with water-air cooling, and for the thickness control - caliper Lascod ZLL010 were used.

A number of scientific articles has alrealdy presented determination of the degree of ligth absorption in biological tissues (in particular into dentin) using discrete light sources (in particular lasers, emitting light on specific wavelength) $[3,4,10$, 11]. Following the inescapable change in the geometric measurement parameters by change of the light source, the absorption determination may be attended with significant errors. Also the fact that the degree of absorption depends on the wavelength is important. In experiments of determination of biological tissue absorption application of light sources with a continuous spectrum of irradiation is advisible. For this reason, in our investigation a xenon lamp with emission spectrum was used as shown in Fig. 1.

The absorption and penetration of light in the wavelength range 350-1000 nm through dentin samples with thickness $1 \mathrm{~mm}$ were measured. Collimated light beam was guided perpendicular to the objects at distance of $5 \mathrm{~mm}$. Immediately behind the tested sample at distance of about 2-3 $\mathrm{mm}$ with the help of lens the passed radiation was focused in flexible quartz optical light guide, which conducted it to the spectrophotometer. Absorption and light transmission of the samples were studied using a 
spectrophotometer Ocean Optics QE65000 and specialized software Spectra Suita Company Ocean Optics (USA) for treating the results. The image of the experimental setup is presented in Fig. 2.

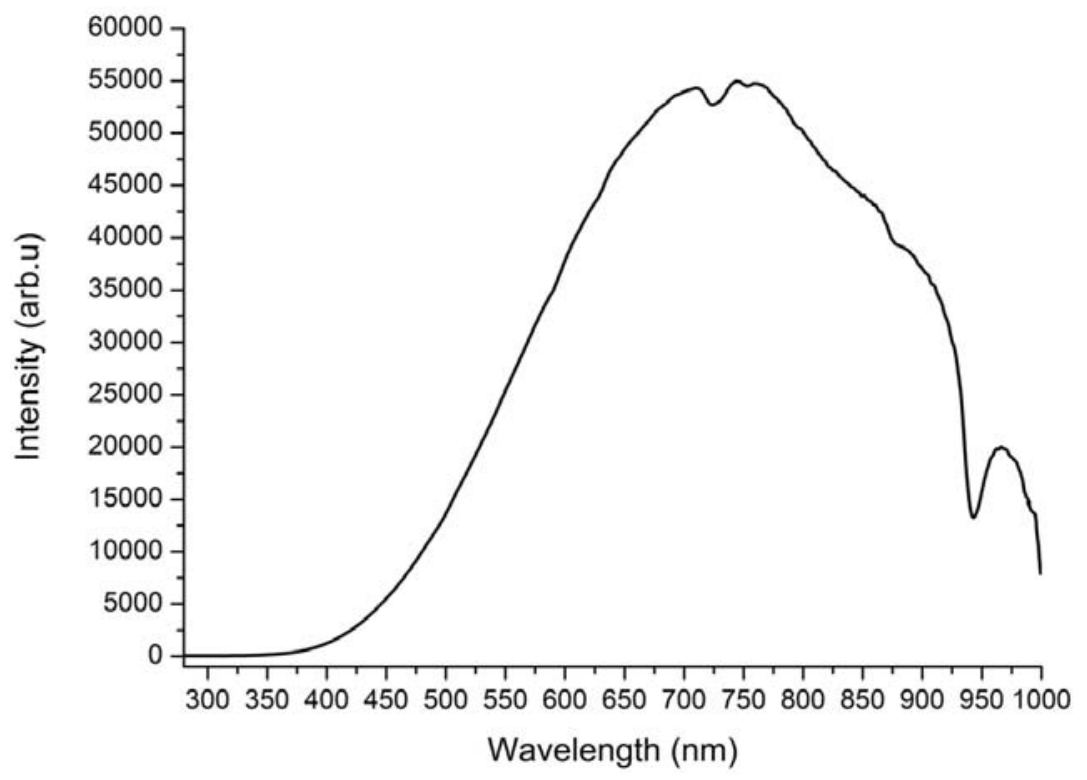

Fig. 1. Emission spectrum of xenon lamp

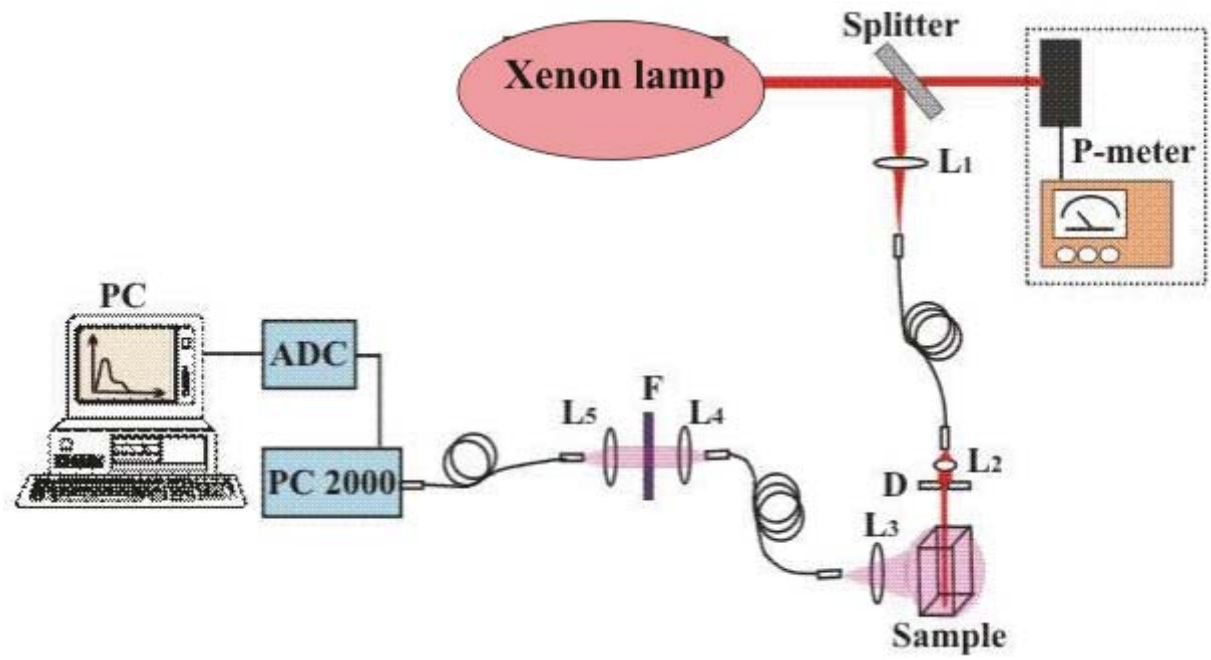

Fig. 2. Experimental setup 


\section{RESULTS}

The results of our study are presented in Fig. 3 and Fig. 4.

Fig. 3 shows image of light absorption depending on the wavelength of 5 tested samples. Statistical analysis of the light absorption results of the remaining 25 tested samples is illustrated with graphics similar to the presented.

Fig. 4 shows image of the average transmission of samples in the studied spectral range.

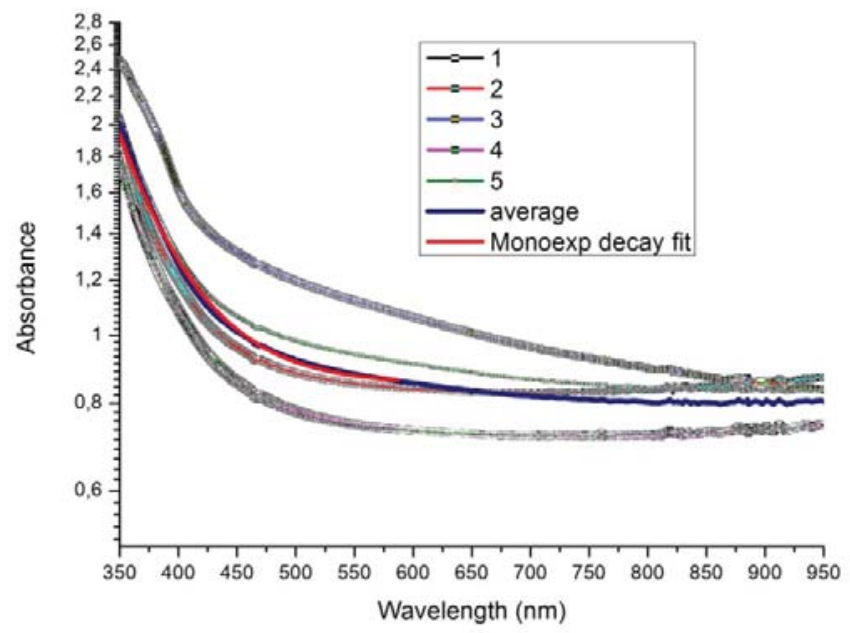

Fig. 3. Absorption of light irradiation of dentin samples, prepared of premolars and molars with $1 \mathrm{~mm}$ thickness

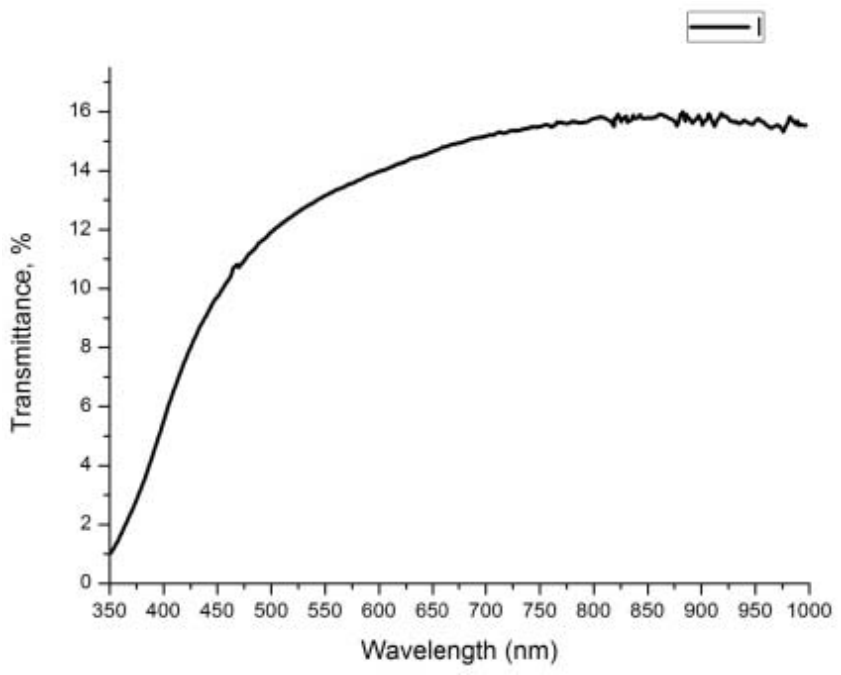

Fig. 4. An average transmission of light radiation in the wavelength range 350-1000 $\mathrm{nm}$ through dentin samples, prepared of premolars and molars with $1 \mathrm{~mm}$ thickness 
The obtained results showed that the light absorption into dentin depends substantially on the wavelength. The relationship between the absorption and the wavelength can be conditionally divided into three areas - from 300 to $600 \mathrm{~nm}$, from 600 to $800 \mathrm{~nm}$ and from 800 to $1000 \mathrm{~nm}$. In UVL (400-315 nm) and the blue-green part of the spectral range, the absorption (with a high degree of reliability) was described by monoexponential decrease, and the penetration was increased. In the area of $600 \mathrm{~nm}$ to $800 \mathrm{~nm}$ a slight decrease in absorption was determined (respectively a slight increase of transmission). In the region above $800 \mathrm{~nm}$ the ratio transmission/ absorption was constant value.

\section{DISCUSSION}

Light penetration depends on the optical characteristics of the tissue, the presence of chromophores, the technical characteristics of the apparatus and the operative technique $[4,6]$. The less the absorption of light with a specific wavelength of the tissue is, the more deeply the light penetrates, therefore penetration increases [6]. Several studies determined that the highest penetration through tissue manifests radiation with wavelength in the near infrared spectral region $(750-1000 \mathrm{~nm})$. The highest absorption into tissues was established in radiation in the blue spectral region [1, $6,10]$. These results were also confirmed in our study. The tooth dentin is composed of organic and inorganic material. The exposure of dentin to a light beamleads to scattering of light from the dentinal tubules. The light of the near- infrared region is poorly absorbed into dentin [2, 10,11].

The observed nature of curves absorption/transmission of dentin can be explained by the presence of natural chromophores, also typical of other biological tissues, and to a minor extent of external pigment factors. Inverse relationship between absorption and penetration of light was described - when absorption increases, permeability decreases. These changes were dependent on the wavelength of the light, which is passed through the dentin samples.

Numerous authors determined that irradiation in the visible spectrum with wavelength between 400 and $600 \mathrm{~nm}$, is absorbed by natural chromophores such as hemoglobin, melanin, etc. The primary chromophore for the infrared region is the water $[6,10]$. In the light spectrum there is a narrow zone of wavelengths, where the water is not highly effective chromophore. This fact allows penetration of light energy to the tissue with high water content. The area (approximately 600 to $1200 \mathrm{~nm}$ ) is called therapeutic window. The most contemporary lasers have wavelength within this therapeutic window [10]. In the present study a configuration of the curve which is characteristic of melanin-like chromophore was obtained. This allows us to state that the absorption of light with a wavelength $600 \mathrm{~nm}$ in dentin is possible and is determined on natural chromophores of its 
composition. It may also be due to bacterial strains, fallen into dentin samples in their preparation of extracted teeth. In the range $600-800 \mathrm{~nm}$ the absorption may be determined on hydroxyapatite, because of its characteristic linear increase of absorption in this area. Above $800 \mathrm{~nm}$ the nature of the curves was determined only on dentin composition, the size of tubular channels, and perhaps therefore the ratio absorption/transmission of dentin samples does not depend on the wavelength from 800 to $1000 \mathrm{~nm}$.

\section{CONCLUSIONS}

The obtained results allow us to make the following conclusions:

1. The penetration of light radiation through dentine samples depends on its wavelength and the absorption of dentin components.

2. The less the absorption of light with specific wavelength into dentin is, the greater the transmission is. That dependence is determined of the light wavelength, which passes through the dentin samples.

3. The greatest penetration into dentin was determined on radiation with wavelength in the near infrared spectral range $(750-1000 \mathrm{~nm})$, and the smallest in UVL $(400-315 \mathrm{~nm})$ and blue-green part of the spectral range. The highest absorption into the tissue was observed at radiation in the blue spectral region, and the least - in the infrared spectral range.

4. When using a light source with a wavelength above $600 \mathrm{~nm}$ and especially in the range $800-1000 \mathrm{~nm}$, the absorption is equal and should be given the intensity of the radiation of source to prevent unacceptable effects.

\section{REFERENCES}

1. Atanasova, E., M.Vasileva, Tz.Uzunov, A.Gisbrecht, M.Nenchev.Oral Tissues Transparency for Laser Radiation from the Visible Spectral Region. - Stomatology, 1986, № 2, 29-33.

2. Bhatia, S., S. Kohli. Lasers in Root Canal Sterilization - a review. - International Journal of Scientific Study, 1, 2013, № 3, 107-111.

3. Dederich, D. Laser tissue interaction. - Alpha Omegan, 84, 1991, 33-36.

4. Dederich, D. Laser/tissue interaction: what happens to laser light when it strikes tissue? - JADA, 124, 1993, № 2, 57-61.

5. Frank, F. Laser light and tissue biophysical aspects of medical laser application. - SPIE Lasers Med, 1353, 1989, 37-45.

6. Gupta, S., S. Kumarbn.Lasers in dentistry - an overview. Trends Biomater. - Artif. Organs, 25, 2011, № 3, 119-123.

7. Gutknech, N., R. Franzen, M. Schippers, F. Lampert. Bactericidal effect of a 980-nm Diode Laser in the root canal wall dentin ofbovine teeth. - J. Clin. Laser Med. Surg., 22, 2004, № 1, 9-13. 
8. Gutknecht, N., A. Moritz ,G. Conrads, G. Lampert. Der dioder laser und seine bacterioizide wirking in wurzerkanal eine in vitro studie. - J. Oral. Laser Appl., 2002, 2, 151-157.

9. Rajeshwari, P. Kamath, M. Kundabala, S. Shenoy, V. Hegde, N. Thukral. An evaluation of horizontal depth of penetration of various irrigants into the dentinal tubules when used alone and in combination with diode laser: an in vitro study. - Journal of Interdisciplinary Dentistry, 4, 2014, № 3, 130-134.

10. Tuner, J., L. Hode. The new laser therapy handbook. - Prima Books AB, Sweden, 2010.

11. Uzunov, Tz., Al. Gisbrecht, M. Nenchev. Investigation of He-Ne laser radiation passing through in teeth incisions. - Problems of Stomatology, 30, 2004, 109-112.

\section{- Corresponding author:}

Assistant professor Violeta Dogandzhiyska

Department Conservative Dentistry, 1020

FDM, MU - Sofia

1 "Sv. G. Sofiiski" St.

e-mail: Dogandzhiyska@gmail.com 